\title{
Analytical validation of an ATR-FTIR based method for quantifying the amount of polysorbate 80 adsorbed on the PLGA nanoparticles
}

Received 00th January 20xx, Accepted 00th January 20xx DOI: $10.1039 / x 0 x \times 00000 x$

\begin{abstract}
Nabodita Sinha, ${ }^{a}$ Abhayraj Shrikrishna Joshi ${ }^{b}$ and Ashwani Kumar Thakur *c
Nanomedicine is envisioned to have a tremendous impact on targeted drug delivery in future. Coating of nanoparticles with non-ionic surfactants has been often employed for enhancing targeting in drug delivery systems. Polysorbate 80 is one of the non-ionic surfactants used often as a coating agent for receptor-mediated endocytosis into the brain. However, very few studies have been done to investigate the actual amount of the surfactant adsorbed or the mechanism of adsorption onto the nanoparticles. We had developed an assay using an ATR-FTIR method for quantiation and adsorption mechanism of polysorbate 80 on PLGA nanoparticles. Here we show the analytical validation of this method, for exploring its suitability for its intended purpose in industries. To comply with regulatory bodies and for standardization, we have followed the ICH and FDA guidelines for analytical validation and investigated the required criteria i.e. accuracy, precision, linearity, range ,limit of detection and quantitation. The method successfully complied with all regulatory criteria and is therefore suitable for successful use in industry, academia and by regulatory bodies.
\end{abstract}

\section{Introduction}

Development of nanoparticles into nanomedicine is expected to ease the drug delivery process due to their unique characteristics and small size. These particles can reach the smallest capillaries or cross obstinate layers such as the bloodbrain barrier. $(1,2)$ Delivering conventional drugs across the blood-brain-barrier has been difficult since it consists of an intricate assembly of endothelial cells and tight junctions which strictly regulates the traffic of molecules from the blood into and out of the brain. (3) Nanoparticle-based drug delivery systems for blood-brain barrier have seen significant growth in research and initial success for their properties such as controlled drug release, and the ability to escape the reticuloendothelial system. These properties increase therapeutic bioavailability in the brain and reduce systemic toxicity. (4) Poly(lactide-co-glycolic) acid- PLGA (FDA approved polymer for biological use) nanoparticles have been exploited for effective drug targeting across the blood-brain-barrier in diseases such as brain tumor and Alzheimer's. The ease of PLGA nanoparticle functionalization, and biodegradability are the major features that make it a suitable candidate for drug delivery. $(5,6)$

Coating of blood brain targeting nanoparticles with a surfactant such as polysorbate 80 is one of the strategies to deliver drug

\footnotetext{
a. Biological Sciences and Bioengineering, IIT Kanpur, India

b. DTU Biosustain, Denmark, Europe.

c. Biological Sciences and Bioengineering, IIT Kanpur, India

+ Footnotes relating to the title and/or authors should appear here.

Electronic Supplementary Information (ESI) available: [details of any supplementary information available should be included here]. See DOI: 10.1039/x0xx00000x
}

molecules to brain in a more effective way. (7) The polysorbate 80 coating enables receptor-mediated endocytosis across the blood-brain barrier by adsorbing to plasma proteins such as apolipoprotein $\mathrm{E}$. The method proved far more efficient as drug delivery system compared to the free drug used. $(8,9)$ Other non-ionic surfactants such as Poloxamer 188 also exhibit similar properties. (10) The concentration of the coated surfactant on the nanoparticles however need to be optimised for efficient and safe drug delivery. While too low amount of surfactant coating can lower the efficacy of the targeting system, (11) high concentration of surfactant on the other hand can result in hypersensitivity reactions in some cases. $(12,13)$ Quantification of the amount of adsorbed surfactant on the nanoparticles thus requires significant attention. However, there have been relatively lower numbers of studies for determining the exact compositional analysis and surfactant concentration present on these particles.

Our lab has previously designed a PLGA nanoparticle system containing polyglutamine aggregation inhibitor peptide $\mathrm{PGQ}_{9}\left[\mathrm{P}^{2}\right]$ for therapeutic approach against Huntington's disease. These nanoparticles were coated with polysorbate 80 for better targeting through the blood-brain-barrier. (18) For designing an optimum drug delivery system and to determine the compositional analysis of the nanoparticles, our lab has recently developed a method for quantifying the exact amount of polysorbate 80 adsorbed onto the PLGA nanoparticles. The method was developed to determine the optimal amount of surfactant adsorbed and also to establish the adsorption mechanism.

In the developed method, empty PLGA nanoparticles (without encapsulated peptides) were prepared by nanoprecipitation 
method and coated with polysorbate 80. For coating, same volume of nanoparticle suspension was mixed with different concentrations of the surfactant. The mixtures were stirred for 0-240 minutes to allow coating at different time points. Before detecting the amount of coated surfactant on the nanoparticles, polysorbate 80 standard curve was prepared. When different amounts of polysorbate 80 was applied on ATRFTIR crystal, the FTIR peak analysis revealed 3 peaks for polysorbate 80 . The $3000-2800 \mathrm{~cm}^{-1}$ peak (acyl group) was selected due to its prominence even at low concentrations. The peak integral area was measured and response for each concentration was measured by calculating the ratio between the polysorbate 80 peak area to an internal standard peak area. After standard curve preparation, a method was developed to optimally extract the coated polysorbate 80 from the nanoparticle surface using repeated washing and centrifugation. The extracted surfactant was then quantified using ATR-FTIR response and by plotting the values in the standard curve. (14)

However, in the drug development to clinical translation efforts, an analytical method or a process needs to be validated for their usage in manufacturing and quality control. International Regulatory agencies such as Food and Drug Administration (FDA) encourages analytical method validation related to the production of any new drug form or for the application of Investigational New Drug (IND). (15) Validation of a developed method provides a degree of assurance that the developed method would prove to be effective and would meet the requirements of its applications. The major advantages of analytical method validation include quality assurance, regulatory compliance, process optimization, and easier scaleup. (16) Analytical validation is based on the following parameters according to the International Conference of Harmonisation (ICH) guidelines: Specificity, Accuracy, Linearity, Range, Limit of Detection, Limit of Quantitation and Precision. (17)

To systematically follow the guidelines for successful translation, we have validated the analytical method for extracting polysorbate 80 and quantifying it by using the ATRFTIR method. All the parameters and workflow have been considered according to the ICH and FDA guidelines.

\section{Experimental Details}

Materials and Equipment Dimethyl Sulfoxide (DMSO) was obtained from Merck. Polyvinyl Alcohol (PVA; Molecular weight 30-70 kDa) and Poly-D,L-lactide-co-glycolide (PLGA) were bought from Sigma Aldrich. Polysorbate 80 and sodium azide both were bought from HiMedia, India. All the DLS experiments were performed on Malvern Zetasizer ZS90 equipped with a 633 $\mathrm{nm}$ laser. Ultracentrifugation for nanoparticle purification was done on Sorvall Microultracentrifuge, ThermoFisher Scientific using Rotor S55-S and S80-AT2. The Attenuated Total Reflection-Fourier Transformed Infra-Red (ATR-FTIR) experiments were performed on Bruker Tensor 27 IR spectrometer (Bruker Optik, Germany) equipped with ATR accessory. The detector used was mercury cadmium telluride (MCT).

\section{Methods}

Preparation of nanoparticles The PLGA nanoparticle preparation and coating were prepared according to the previously established protocols using the nanoprecipitation method. (18) For each batch of nanoparticle preparation, $15 \mathrm{mg}$ of Polylactide-co-glycolide (PLGA) was weighed in an empty microcentrifuge tube of $2 \mathrm{ml}$ volume. The weighed powder was dissolved in $1 \mathrm{ml}$ of Dimethyl sulfoxide (DMSO) by vortexing for 5-6 minutes. After ensuring by visual inspection that all PLGA had dissolved, the PLGA solution was transferred to a $5 \mathrm{ml}$ syringe pump and care was taken to remove any bubbles which might appear during transfer. $10 \mathrm{ml}$ of $3 \%$ Polyvinyl alcohol (PVA) solution (in water) was prepared in a $15 \mathrm{ml}$ glass vial. The syringe with the PLGA solution was placed in the infusing pump and set in place. The pump was set at a flow rate of $120 \mathrm{ml} / \mathrm{h}$ and infusion was started. After infusion, the vial with the suspension mixture was rotated at $700 \mathrm{rpm}$ for 30 minutes more.

The suspension from the vial was transferred to evaporator flask using $1 \mathrm{ml}$ pipette. The flask was fitted to the rotary evaporator and the DMSO was then evaporated from the mixture in a rotary vacuum evaporator $\left(40^{\circ} \mathrm{C}, 3-4\right.$ hours, 50 $\mathrm{rpm})$. After evaporation, the remaining suspension of about 8$9 \mathrm{ml}$ was distributed in 4 microcentrifuge tubes. The nanoparticle suspension was then subjected to two cycles of centrifugation to remove excess PVA. The first centrifugation was at $90000 \mathrm{~g}(32400 \mathrm{rpm})\left(\mathrm{RCF}=1.12 \times \mathrm{R} \times(\mathrm{RPM} / 1000)^{2}\right.$ where $R$ is the radius of the rotor $S-55 S, 76.55 \mathrm{~mm}$ ) for 50 minutes. The supernatant was discarded and the pellets were resuspended in distilled water. The second centrifugation was at $60000 \mathrm{~g}$ (22000 rpm) for 10 minutes. The pellets obtained were resuspended in $4 \mathrm{ml}$ of distilled water and vortexed. Before coating, we analyzed $1 \mathrm{ml}$ of the nanoparticle suspension by DLS method to check z-average hydrodynamic diameter, polydispersity index (PDI), and zeta potential. All DLS measurements were performed after diluting the nanoparticle suspension to prevent multiple light scattering. For this, $50 \mu \mathrm{L}$ of nanoparticle was diluted with $950 \mu \mathrm{L}$ of double distilled water.

For determining the mass of nanoparticles produced, $1 \mathrm{ml}$ of the nanoparticle suspension was transferred to a pre-weighed tube and dried in a vacuum evaporator until fully dry. The weight of the tube was again recorded. The mass of the nanoparticles was recorded as: (Final Weight-Tube Weight) for $1 \mathrm{ml}$ suspension.

Coating PLGA nanoparticles with polysorbate 80 A stock solution of polysorbate 80 of concentration $200 \mathrm{mg} / \mathrm{ml}$ and 50 
$\mathrm{mg} / \mathrm{ml}$ were prepared. For coating of the nanoparticles, $1 \mathrm{ml}$ of nanoparticle suspension was mixed with $50 \mathrm{mg} / \mathrm{ml}$ or 200 $\mathrm{mg} / \mathrm{ml}$ solution and final volume was made up to $1250 \mu \mathrm{l}$ with water to prepare the final concentration of polysorbate 80 as $0.1,0.3,0.5,1,3,5,7.5,10,20$ and $30 \mathrm{mg} / \mathrm{ml}$. The coating was performed by simply stirring the nanoparticle-surfactant mixture at $100 \mathrm{rpm}$ for 0-240 minutes. (14) (ESI Table S1) At each time point $(0,20,30,60,120,240$ minutes $)$, the coated nanoparticles were characterized using Dynamic Light Scattering. The hydrodynamic diameter, zeta potential and polydispersity index values were obtained for each sample.

Preparation of polysorbate 80 standard curve Using a stock of 0.5 $\mathrm{mg} / \mathrm{ml}$ of polysorbate $80,2 \mathrm{ml}$ of the following dilutions was prepared: $0.3125,0.625,1.25 .2 .5,5,7.5,10,15,20,30$ and 40 $\mu \mathrm{g} / \mathrm{ml}$. $50 \mu \mathrm{l}$ of each dilution was then mixed with $1 \mu \mathrm{l}$ of $1 \%$ sodium azide (internal standard). For each concentration, the amount of polysorbate 80 in $\mu \mathrm{g}$ was calculated. These samples were immediately analyzed by ATR-FTIR under continuous nitrogen gas purging. $1 \mu \mathrm{l}$ of each of the prepared samples was placed on the liquid nitrogen-cooled zinc-selenide crystal of the ATR unit. In $1 \mu$ lof the sample, the amount of polysorbate 80 was calculated and expressed in $\mathrm{ng}$. Thus the following amounts were used per $\mu \mathrm{l}$ for standard curve: $0.3125,0.625,1.25 .2 .5,5$, $7.5,10,15,20,30$ and $40 \mathrm{ng}$. Water was scanned as background (120 scans. $4 \mathrm{~cm}^{-1}$ ) before each sample analysis.

For each sample, 60 scans were recorded the range of 4000 $850 \mathrm{~cm}^{-1}$. Scan resolution was $4 \mathrm{~cm}^{-1}$. The primary spectra obtained were opened using OPUS software and were baseline corrected using the Rubberband baseline correction method (64-point format). The spectra were smoothened using 17 smoothening points. The Integral Area Function of OPUS was used to calculate the area of the peaks. For azide (internal standard) and acyl (polysorbate 80 ) peaks, 2080-1970 cm-1 and $3000-2800 \mathrm{~cm}-1$ regions were used as signature peaks. For preparing the standard curve, the ratio of the integrated acyl peak area of the polysorbate 80 to the azide peak area (internal standard) was plotted against the concentration of polysorbate 80 in the sample. After plotting a scattered plot, the linear fit was established by using Origin Pro 9.1 software using Analysis>Linear Fit option.

Extraction of polysorbate 80 from the nanoparticles and quantification: The extraction of the surfactant from the nanoparticles was done by repeated dissolution and centrifugation method as described previously. (14) $150 \mu \mathrm{L}$ nanoparticle samples were collected after 240 minutes of coating and ultracentrifugation was done at $25000 \mathrm{~g}$ for 4 minutes to remove the excess of polysorbate 80 . The supernatant was discarded. The pellets containing coated nanoparticles were washed with $150 \mu \mathrm{L}$ water and the second cycle of centrifugation at $25000 \mathrm{~g}$ for 4 minutes was done. The pellets obtained were dried under vacuum desiccator for 2 hours. The desiccator was run at $45^{\circ} \mathrm{C}$. The dried pellets were dissolved in $150 \mu \mathrm{L}$ acetonitrile. This precipitates the PVA which can then be removed by ultracentrifugation at $25000 \mathrm{~g}$ for 30 minutes. The mixture containing PLGA, polysorbate 80 , and acetonitrile was then vacuum evaporated $\left(45^{\circ} \mathrm{C}\right)$ for 2 hours for removing acetonitrile and thus only PLGA and Polysorbate 80 remained. Polysorbate 80 which is soluble in water was then removed by ultracentrifugation at $150000 \mathrm{~g}$ for 2 hours (Rotor S80 AT2) as PLGA precipitated out. The clear supernatant containing polysorbate 80 was collected. To $50 \mu \mathrm{L}$ of the supernatant sample, $1 \mu \mathrm{L}$ of $1 \%$ sodium azide was added. $1 \mu \mathrm{L}$ of these prepared samples were quantitated by ATR-FTIR as already described above.

The ratio of the polysorbate peak to azide peak was plotted in the standard curve to get the amount of surfactant extracted from the nanoparticles as $\mu \mathrm{g}$. The amount was then divided by the mass of PLGA nanoparticles ( $\mathrm{mg}$ ) to get the adsorbed surfactant amount as ( $\mu \mathrm{g} / \mathrm{mg})$.

Validation Criteria: According to the $\mathrm{ICH}$ guidelines, the following criteria are required for analytical validation of an assay: Specificity, Accuracy, Reproducibility, Precision, Linearity, Range, Limit of Quantification, and Detection. The specificity of the method has been established in our method development. The interfering signals in FTIR may be contributed by PLGA degradation products such as lactic acid and glycolic acid or residual PVA present on the nanoparticles. The FTIR peaks exhibited by these molecules were recorded. PVA shows sharp FTIR peaks at 2935 and $1750 \mathrm{~cm}^{-1}$. Lactic acid shows multiple peaks between $2850-2980 \mathrm{~cm}^{-1}$ and $1700-1300 \mathrm{~cm}^{-1}$. Glycolic acid shows peaks at 2919 and $1595 \mathrm{~cm}^{-1}$. The samples after extraction of polysorbate 80 from nanoparticles were then analysed by FTIR and the presence of these interfering signals were investigated. Since one set of the signals for each of these molecules falls within the acyl peak range of $3000-2800 \mathrm{~cm}^{-1}$ of polysorbate 80 , the other signatory peaks were used as markers, i.e. for PVA at $1750 \mathrm{~cm}^{-1}$, for lactic acid at 1700-1300 $\mathrm{cm}^{-1}$ and for glycolic acid $1595 \mathrm{~cm}^{-1}$. None of the samples showed the presence of signature peaks of PVA or PLGA degradation products. (14) In method validation also, the extracted polysorbate 80 samples were recorded in FTIR and the interfering signals if any were investigated. We determined the accuracy of the method by preparing 3 known concentrations of polysorbate 80 i.e. 4,_ 8 and $12 \mathrm{ng} / \mu \mathrm{L} .1 \mu \mathrm{L}$ of these samples were then analyzed by ATR-FTIR and the obtained experimental values were compared to the true values. Accuracy \% was calculated by the following formula:

Accuracy $\%=[\{($ Actual value $-($ Actual value - Measurement $)\} /$ Actual value] $\times 100$

The precision and reproducibility were analyzed by measuring the relative standard deviation between the extracted polysorbate 80 amounts obtained after 240 minutes of coating on different days. For this, the limit of extracted surfactant at the last time-point i.e. 240 minutes was compared to observe whether the relative standard deviations are within the acceptable limit. The linearity and range were determined from 
the standard curve. The linearity is determined by the values of the standard curve for which the response increases in a proportional manner. The start and endpoint of this linearity is denoted as the range.

The lowest values which could be detected and quantified considering acceptable accuracy and precision using the developed method were defined as the LOD and LOQ respectively. These values were also calculated using the slope and regression values of the calibration curve as described in the $\mathrm{ICH}$ guidelines. LOD can be defined using the following formula $\mathrm{DL}=(3.3 \times \sigma) / \mathrm{S}$ where $\mathrm{DL}$ is the detection limit, $\mathrm{S}$ is the slope of the response and $\sigma$ is the standard deviation of the response. LOQ can be determined according to the formula $L O Q=10(\sigma / S)$.

\section{Results}

Characterization of the nanoparticles: The PLGA nanoparticles prepared and coated according to the above-mentioned methods were characterized by Dynamic Light Scattering procedure which determines the average hydrodynamic diameter of these nanoparticles. The coated nanoparticles were analysed for the hydrodynamic diameter at different time points from 5 minutes to 240 minutes of coating to infer the effect of coating on the size of the nanoparticles. The DLS data shows that the coated nanoparticles do not show any significant increase or decrease in size as compared to the uncoated nanoparticles (Figure 1 a). The hydrodynamic diameter was found to be $150 \pm 9.87 \mathrm{~nm}$. The polydispersity index was $0.045-$ 0.055 which suggests that the coated nanoparticles are stable in suspension and do not form aggregates after coating (Figure $1 \mathrm{~b})$. The zeta potential was found to be $-22.4 \pm 4.3$ milliVolts indicating moderately stable suspension. (ESI Table S2)

In our method development, the hydrodynamic diameter of the PLGA nanoparticles (without encapsulated peptides) was found to be $\sim 156 \pm 7.83 \mathrm{~nm}$. The polydispersity index range was $0.039 \pm 0.019$. (14) The results show that both method development and validation yield similar hydrodynamic diameter and polydispersity index. This signifies that the developed nanoprecipitation method is a suitable and validated process for preparing $150 \pm 10 \mathrm{~nm}$ stable PLGA nanoparticles coated with polysorbate 80 with PDI $0.05 \pm 0.03$. The zeta potential of the particles was found to be $-27.9 \pm 4.92$ milliVolts during method development which again indicates moderately high stability.

The characterization and validation of these coated nanoparticles are essential because literature shows that the concentration of the surfactant present in the system can have an effect on the size of the nanoparticles. The hydrodynamic diameter can decrease or increase with increase in the surfactant concentration. (19)_In some cases, the hydrodynamic diameter of the nanoparticles has been also observed to influence the morphology and adsorption properties of the surfactant molecules. (20) Our prepared nanoparticles show similar specification parameters of hydrodynamic diameter, polydispersity index and zeta potential at both development and validation stage with no statistically significant difference (t-test, $p>0.05$ ).

As a process parameter, the nanoprecipitation yield was also calculated. For this, $1 \mathrm{ml}$ of the nanoparticle suspension was dried in a pre-weighted tube and the weight of the dried tube was recorded. The mass difference by evaporating and weighing method was found to be $\sim 2.24 \mathrm{mg}$ for $1 \mathrm{ml}$ nanoparticle solution. In a single batch of PLGA nanoparticle production, 15 $\mathrm{mg}$ of PLGA is used which forms $4 \mathrm{ml}$ of nanoparticle suspension. Thus total weight of nanoparticle in $4 \mathrm{ml}$ of suspension would be $(2.24 \times 4) \mathrm{mg}$. The yield\% was thus $\{(2.24 \times 4) / 15\} \times 100=59.7 \pm 6.1 \%$. During method development, the yield\% was $62.96 \pm 6.29 \%$ and is not significantly different from the validation yield ( $t$-test, $p>0.05$ ).
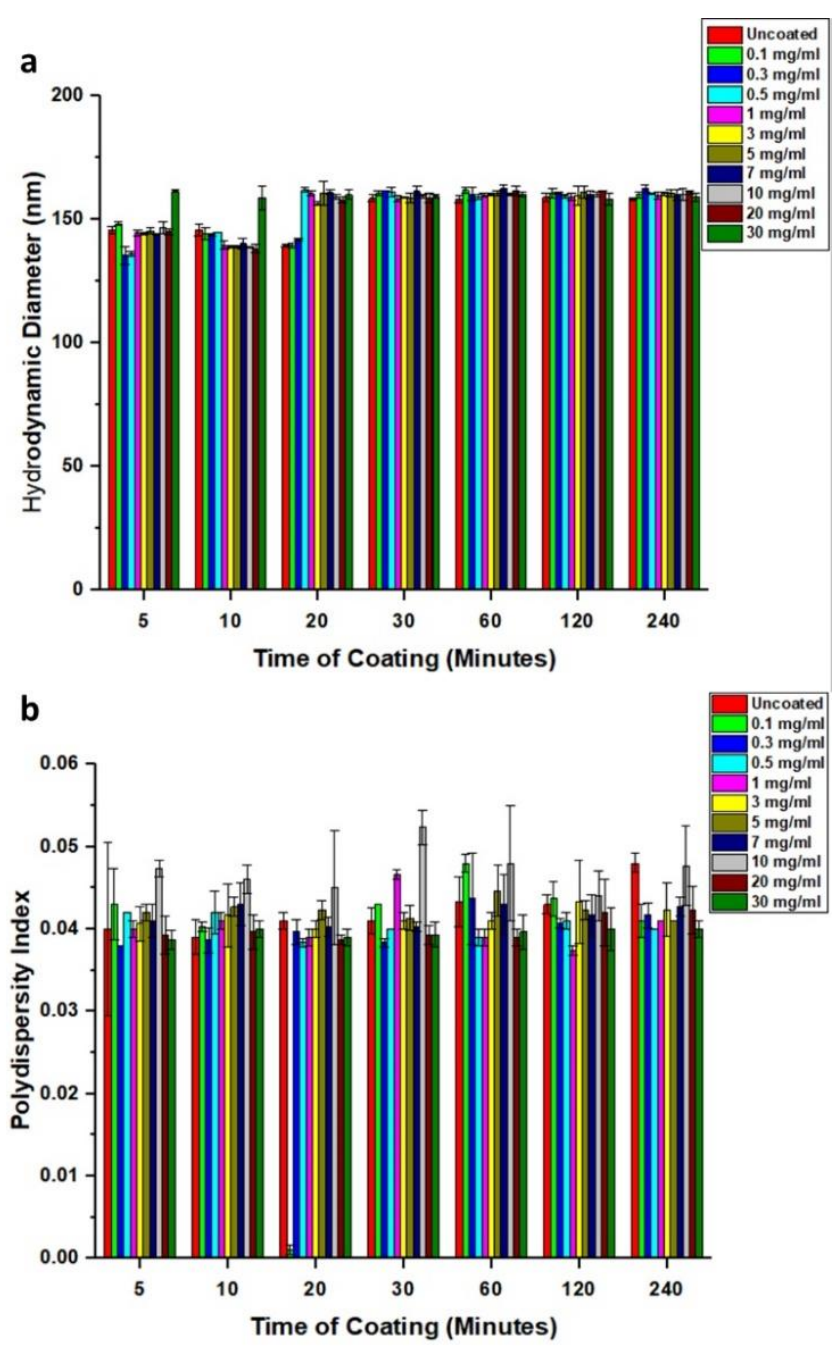

Figure 1. The hydrodynamic diameter of the coated vs. uncoated nanoparticles at each time point and concentration of surfactant coating shows no significant change in the size of the nanoparticles after coating; b. low polydispersity index around 0.05 suggests that the nanoparticles are well-dispersed and homogeneous in suspension. 
Standard Curve of Polysorbate 80 and determination of linearity, range, Limit of Detection and Quantitation: For any analytical validation method, the calibration curve is important, because from this graph, parameters i.e. linearity, range, Limit of Detection (LOD) and Limit of Quantitation (LOQ) can be calculated. For developing the calibration curve of Polysorbate $80,1 \mu \mathrm{L}$ sample each containing the amounts of 0.3125 to $40 \mathrm{ng}$ were analyzed by FTIR. Polysorbate 80 peaks were seen at 3000$2800 \mathrm{~cm}^{-1}$ (acyl peak), 1750-1850 cm-1 (ester peak) and 1050$1150 \mathrm{~cm}^{-1}$ (ethoxy peak). However, among the three IR peaks, the $3000-2800 \mathrm{~cm}^{-1}$ peak was chosen for the method development due to its sharp and easily detectable nature even at very low concentrations (18). The other two peaks are detectable only at higher concentrations and therefore were not chosen for standard curve. Sodium azide $(1 \mathrm{mg} / \mathrm{ml})$ was chosen as the internal standard which gives sharp peaks at 2080-1970 $\mathrm{cm}^{-1}$, at significantly different position from the polysorbate 80 peaks. The internal standard is essential to minimize errors between readings and to minimize variations due to internal conditions.

After obtaining the IR spectra for each amount of polysorbate 80 , the ratio of peak area of polysorbate 80 to sodium azide was used to plot the standard curve against the amount of polysorbate 80 used. As shown in Figure $\mathbf{2}$ a, the FTIR primary stacks show the polysorbate 80 and sodium azide peaks for increasing amount of polysorbate 80 . For each amount, the FTIR response was calculated as the ratio of polysorbate 80 to sodium azide peak area. The integral peak area was calculated by using Opus Software. These ratios were then plotted against the amount of polysorbate 80 for standard curve. Figure $\mathbf{2} \mathbf{b}$ shows the calibration curve obtained after analyzing polysorbate 80 dilutions. The linear regression curve is fitted using Origin Pro 9.1 software. (Methods: Preparation of polysorbate 80 standard curve) The equation obtained is $y=0.41236 x+0.17651$. The curve shows a good fit of $r^{2}=0.99852$.

From the calibration curve, the LOD was found to be $0.625 \mathrm{ng}$ below which we didn't detect any peak for the acyl group of polysorbate 80 . However as evident from observing the standard curve, the LOQ is found to be $1.25 \mathrm{ng}$. Below this value, the samples could not be quantified. The upper value up to which the results were obtained in proportion to the amount was $20 \mathrm{ng}$. Above this value, any increase in area of the acyl peak was not observed. The linearity and range of the curve was thus found to be between 1.25 to $20 \mathrm{ng}$. In our method development, the calibration curve had similar linearity and range values of 1.25 to $20 \mathrm{ng}$. This exhibits the high sensitivity of the ATR-FTIR method since the measurements are reproducible at nanoscale.

Using the $\mathrm{ICH}$ formula $(\mathrm{DL}=(3.3 \times \sigma) / \mathrm{S}$ where $\mathrm{DL}$ is the detection limit, $S$ is the slope of the response and $\sigma$ is the standard deviation of the response), the LOD was calculated. From the curve equation, the slope is 0.41236 . The regression analysis shows that the $\sigma$ value is 0.07591 . Thus $\mathrm{DL}=$ $(3.3 \times 0.07591) / 0.41236=0.60748 \mathrm{ng}$. This value closely matches with that calculated manually LOD value of $0.625 \mathrm{ng}$. LOQ can be determined according to the formula $L O Q=10(\sigma / \mathrm{S})$. Thus using our calibration curve, LOQ would be = $10(0.07591 / 0.41236)=1.8 \mathrm{ng}$. This value also corresponds closely to our manually observed value of LOQ i.e. $1.25 \mathrm{ng}$. In our method development, for samples in solution state, lowest amount that could be detected and quantified was $1.25 \mathrm{ng}$. The LOQ values in both method development and validation are similar (1.25 ng). However, the LOD values vary slightly signifying the fact that the detection level may vary from (0.6 to $1.25 \mathrm{ng})$
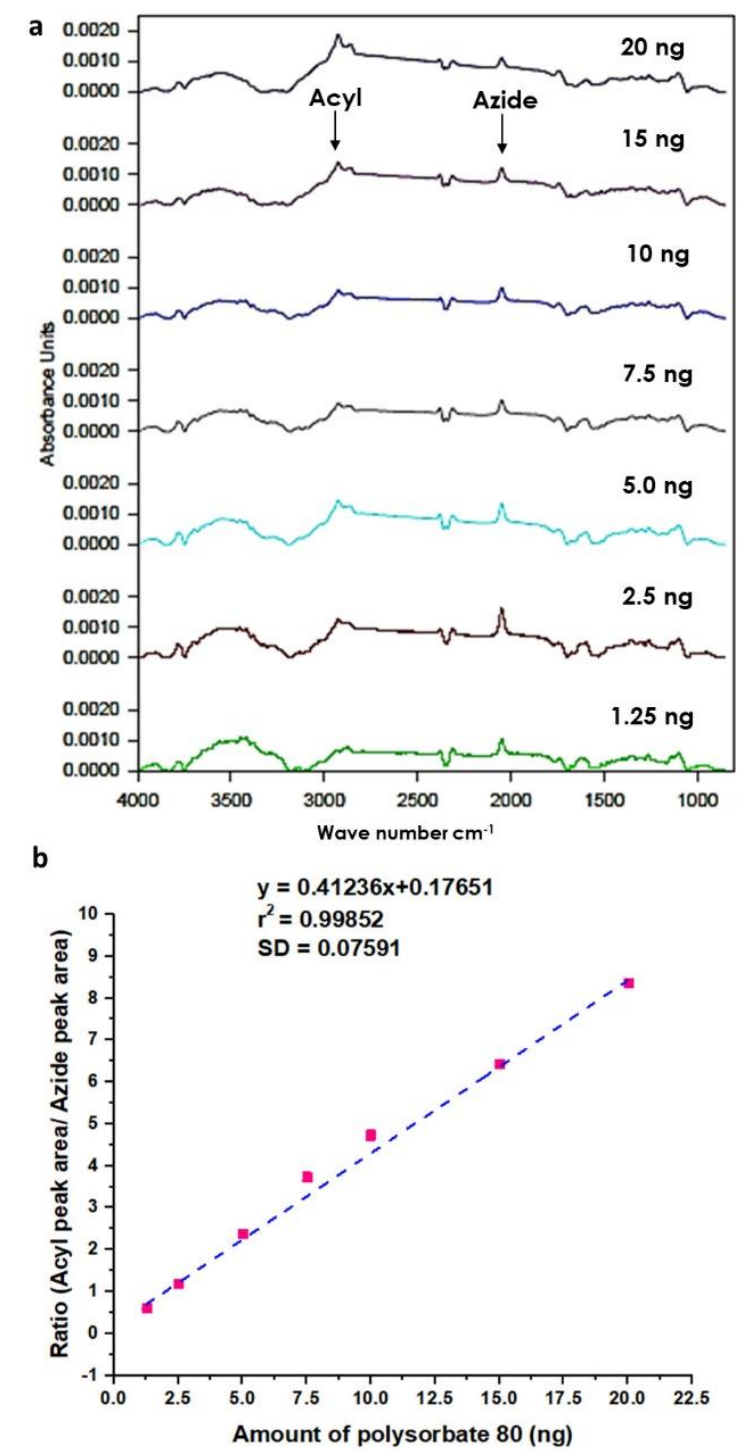

Figure $2 \mathrm{a}$. The FTIR primary spectra of different amounts of polysorbate 80 . The acyl peak of polysorbate 80 is evident around $2800-2900 \mathrm{~cm}^{-1}$ and the azide peak of the internal standard is found around $2100-2200 \mathrm{~cm}^{-1} ; \mathrm{b}$. The calibration curve obtained from standard concentrations of polysorbate 80 showing a good fit of the linear regression of 0.99852 . The standard deviation of the regression is 0.07591 with a slope 
Accuracy Determination: This parameter is used to check how reliable or predictable the standard curve is for calculating an unknown concentration. For checking the accuracy of the developed method, 3 samples of polysorbate 80 were prepared at known amount i.e. 4, 8, and 12 ng by diluting the stock sample of $50 \mathrm{mg} / \mathrm{ml}$. These amounts were selected based on the guidelines that these should be within the linearity and range of the calibration curve but should not coincide with the specific points taken for preparing the curve.

These samples were then analyzed by FTIR and the ratio of the peaks calculated. These ratios were then plotted on the standard curve to calculate the experimental concentration. The accuracy $\%$ is then calculated according to the following formula: Accuracy \% = ((Actual value - (Actual value Measurement)) / Actual value) $\times \mathbf{1 0 0}$

The accuracy \% of all three samples vary between $96 \%$ to $99 \%$ showing that the method is indeed accurate. Figure $\mathbf{3}$ a shows the accuracy of the three samples.

Extraction of the Polysorbate $\mathbf{8 0}$ from the nanoparticles and quantification - The surfactant was extracted from the nanoparticles using the method developed in our lab earlier. For each concentration, the coating of PLGA nanopartciles was done for 240 minutes and then the samples were processed to remove the PVA and PLGA which gives interfering signals in FTIR. These samples were then analyzed by FTIR in the liquid state and the ratio of the peak areas was obtained from the graphs and plotted on the standard curve to get the amount of extracted surfactant.

Figure $3 \mathrm{~b}$ shows the stacked primary spectra obtained at different time points after coating with $0.3 \mathrm{mg} / \mathrm{ml}$ of polysorbate 80 . To further check whether the extracted samples had any PVA left, these were further analyzed by the Ellamann Method. The percentage of the PVA present in these samples was found to be $1.2 \pm 0.4 \%$. This residual PVA did not exhibit any peaks in the FTIR and thus further demonstrates the speciifcity of the method.

For obtaining the precision level of the developed method, first the repeatability test was performed. This criteria checkes whether there are significant statistical difference in the analysis of the extracted samples when performed on the same day (intraday precision). For this, 3 sets of extracted samples were prepared for each concentration, and analysis was done (3 concentrations each for 3 replicates). For intermediate precision, reproducibility was assayed. Reproducibility is established when the extractions and analysis are performed on different days (interday precision) or by different analysts. For this, the entire set of experiments was repeated on different days (3 replicates). At each concentration, the extraction values obtained are shown in ESI Table S3. ESI Table S4 shows the \%RSD values expressing the degree of precision. The comparative results are depicted graphically in Figure 4. The $\%$ RSD values are within the range of $0-20 \%$ for intermediate precision level.
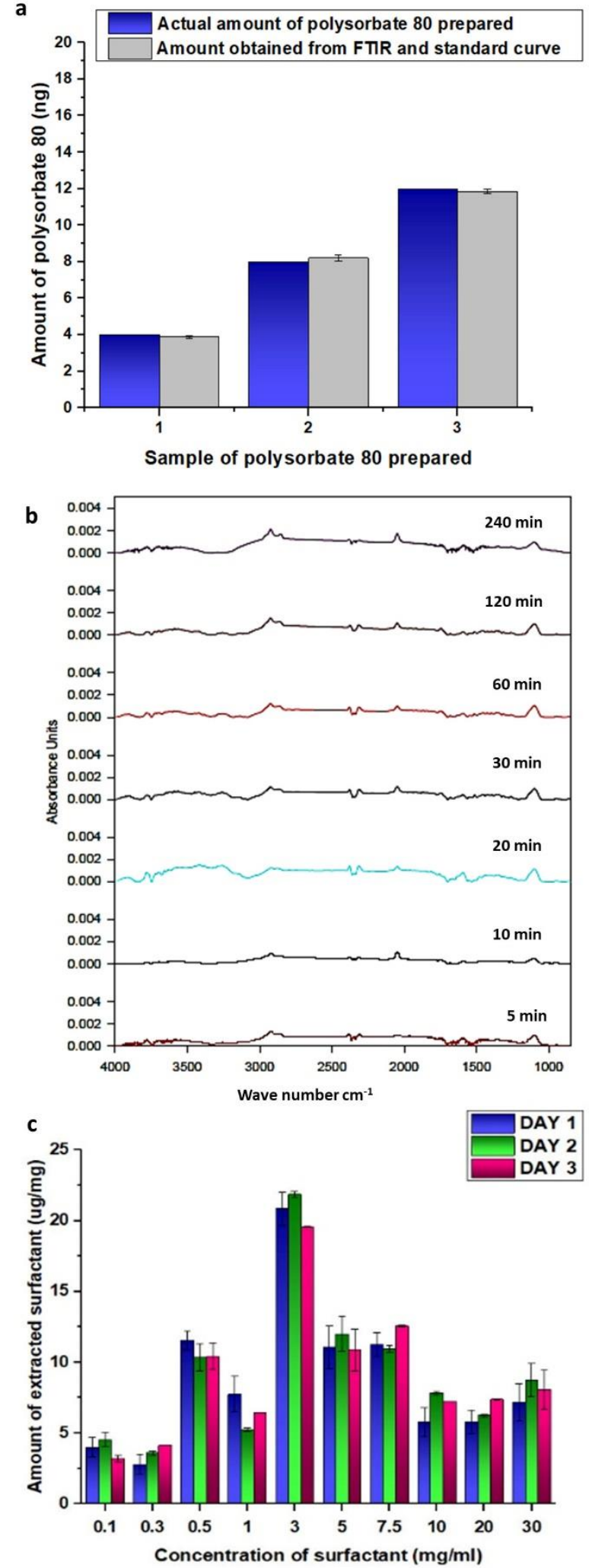

Figure 3 a. The closeness between true values and determined values show that the method is highly accurate. b. The FTIR primary spectra obtained for samples of extracted surfactant after each time intervals. c. Intermediate Precision test followed by repeating the analysis on different days. 
In summary, the developed method shows high accuracy of 96 $99 \%$. The linearity and the range of the method are within 1.25 to $20 \mathrm{ng}$. The LOD of the method is within 0.607 to $0.625 \mathrm{ng}$. The LOQ falls within 1.25 to $1.8 \mathrm{ng}$. The method is reproducible when performed on different days and thus complies intermediate precision.

Table 1: Process Development and Validation Parameters

\begin{tabular}{|c|c|c|}
\hline Parameter & Develeopment & Validation \\
\hline $\begin{array}{c}\text { Nanoparticle } \\
\text { Hydrdynamic } \\
\text { Dimater }\end{array}$ & $156 \pm 7.83 \mathrm{~nm}$ & $150 \pm 9.87 \mathrm{~nm}$ \\
\hline $\begin{array}{c}\text { Polydispersity } \\
\text { Index }\end{array}$ & $0.039 \pm 0.019$ & $0.05 \pm 0.005$ \\
\hline Zeta Potential & $-27.9 \pm 4.92 \mathrm{mV}$ & $-22.4 \pm 4.3 \mathrm{mV}$ \\
\hline Residual PVA & $0.9 \pm 0.3 \%$ & $1.2 \pm 0.4 \%$ \\
\hline $\begin{array}{c}\text { Nanoprecipitation } \\
\text { Yield }\end{array}$ & $62.96 \pm 6.29 \%$ & $59.7 \pm 6.1 \%$ \\
\hline Linearity & $1.25-20 \mathrm{ng}$ & $1.25-20 \mathrm{ng}$ \\
\hline LOD & $1.25 \mathrm{ng}$ & $0.607-0.625 \mathrm{ng}$ \\
\hline LOQ & $1.25 \mathrm{ng}$ & $1.25 \mathrm{ng}$ \\
\hline & & \\
\hline
\end{tabular}

\section{Conclusions}

Our lab has previously designed PLGA nanoparticles coated with polysorbate 80 for delivering peptide-based inhibitors across blood-brain-barrier as a therapeutic approach for Huntington's disease. For designing an optimal drug delivery system, we also wanted to analyse the amount of the surfactant coated onto the nanoparticles. Our lab developed an ATR-FTIR based method in our laboratory which can detect polysorbate 80 surfactant extracted from PLGA nanoparticles at a nanogram scale. For any developed method which is involved with a drug development or drug delivery, analytical validations are an integral component. Validations are encouraged by the international regulatory guidelines for any new drug applications and methods associated with it since it provides confidence regarding the method's reproducibility. Even for preparing a monograph, analytical validations are essential.

In this regard, we analytically validated the ATR-FTIR method following the ICH and FDA guidelines. We found the method to have a high accuracy of $96-99 \%$. The linearity and range of the method are found to be within 1.25-20 ng. The LOD and LOQ values are as low as $\sim 0.607$ and $\sim 1.25 \mathrm{ng}$ respectively. The method complied with intermediate precision and reproducibility when performed on different days. The extraction of the surfactant after 240 minutes of the coating was found to have a relative standard deviation of 0-20\%. For further implementations or technology transfer in the near future, the method would be approved by a regulatory agency.
Although analytical validation is prevalent in the industries, very few research labs follow this path for validating their processes, and thus in the long-run leads to the failure of the processes on a large scale. The systematic workflow can also be used to validate similar analytical methods where quantification of adsorbed molecules on nanoparticles need to be quantified for optimum drug delivery design system.

\section{Conflicts of interest}

There are no conflicts to declare.

\section{Acknowledgements}

The acknowledgements come at the end of an article after the conclusions and before the notes and references.

\section{References}

1 Pehlivan SB. Pharm Res , 2013, 30, 2499-2511.

2 Kanwar JR, Sriramoju B, and Kanwar RK, 2012; 7: 3259-3278.

3 Harilal S, Jose J, Parambi DGT, Kumar R, Unnikrishnan M.K., Uddin S, Mathew G.E., Pratap R, Marathakam A and Mathew,,Brain Res Bull. 2020, 160, 121-140.

4 Teleanu D.M., Chircov C, Grumezescu A.M., Volceanov A, and Teleanu R.I., Pharmaceutics, 2018,10(4),269.

5 Mondal J., Patra M., Panigrahi A.K., and Khuda-Bukhsh A.R, J. Ayurveda Integr. Med., 2020, 11(1), 24-36.

6 Malinovskaya Y., Melnikov P., Baklaushev V., Gabashvili A., Osipova N., Mantrov S., Ermolenko Y., Maksimenko O., Gorshkova M., Balabanyan V., Kreuter J and Gelperina S, Int. J. Pharm., 2017, 524, 77-90.

7 J. Kreuter, P. Ramge, V. Petrov, S. Hamm, S.E. Gelperina, B. Engelhardt, R. Alyautdin, H. von Briesen, and D.J. Begley, Pharmaceut. Res. , 2003, 20, 409-416.

8 J. Kreuter, D. Shamenkov, V. Petrov, P. Ramge, K. Cychutek, C. Koch-Brandt, and R. Alyautdin, ,J. Drug Target. 2002, 10, 317325.

9 J. Kreuter, J. Nanosci. Nanotechnol. , 2004, 4, 484-488.

10 B. Petri, A. Bootz, A. Khalansky, T. Hekmatara, R. Müller, R. Uhl, J. Kreuter, and S. Gelperina, J. Control. Release, 2007, $117,51-58$.

11 J. Kreuter, D. Shamenkov, V. Petrov, P. Ramge, K. Cychutek, C. Koch-Brandt and R. Alyautdin, J. Drug Targeting, 2002, 10, 317 $-325$.

12 Schwartzberg LS, Navari RM. Adv Ther. , 2018, 35(6):754-767.

13 Coors EA, Seybold H, Merk HF, and Mahler V. Ann Allergy Asthma Immunol. 2005, 95(6), 593-599.

14 Joshi, A. S., Gahane A, and Thakur A.K. RSC Advances, 2016, 6(110), 108545-108557.

15 Analytical Procedures and Methods Validation for Drugs and Biologics, 2015, US FDA. Docket Number: FDA-2015-N-0007; https://www.fda.gov/regulatory-information/search-fdaguidance-documents/analytical-procedures-and-methodsvalidation-drugs-and-biologics.

16 Lavanya G, Sunil M, Eswarudu M.M., Eswaraiah M.C., Harisudha K, and Spandana B.N. IJPSR, 2013; 4(4): 1280-1286.

$17 \mathrm{ICH}$ Harmonised Tripartite Guideline: Validation of Text and Methodology, Q2(R1), 2005; https://database.ich.org/sites/default/files/Q2_R1__Guideli ne.pdf

18 Joshi, A.S. and Thakur, A.K. J. Pept. Sci., 2015, 20, 630-639.

19 Martínez-Gutiérrez, H., et al. 2009; Macromolecular Symposia 283-284(1): 34-44. 
20 Lugo, D. M., et al. The Journal of Physical Chemistry B, 2018 114(12): 4183-4191. 